\title{
LENS - Laser Energy for Nuclear Science facility @ LNS
}

\author{
Carmen Altana ${ }^{1, *}$, Gaetano Lanzalone ${ }^{1,2}$ Annamaria Muoio $^{3}$, and Salvatore Tudisco ${ }^{1}$ \\ ${ }^{1}$ INFN - LNS, Via S. Sofia 62, 95123 Catania, Italy \\ ${ }^{2}$ Univ. degli Studi di Enna "Kore", Via delle Olimpiadi, 94100 Enna, Italy \\ ${ }^{3}$ IMM-CNR, VIII Strada, 5, 95121 Catania, Italy
}

\begin{abstract}
A dedicated laboratory at Laboratori Nazionali del Sud (LNS) of Catania was realized with the aim to investigate nuclear reactions of astrophysical interest in plasma environment. In this contribution, the facility LENS (Laser Energy for Nuclear Science), useful to study laserproduced plasmas, will be described and some results will be presented.
\end{abstract}

\section{Introduction}

The $99 \%$ of the matter in the Universe is plasma, which has a primary role in modern astrophysics. Therefore, the investigation of the origin of stars and related phenomena dials closely with plasma physics. Moreover, the measurements of nuclear reaction rates and theoretical estimations in order to understand the origin of the chemical elements and the energy generation in stars is possible thanks to Nuclear Astrophysics [1-3]. For this reason, the study of laser produced plasmas could be important for nuclear astrophysics implications [4].

Moreover, the availability of extremely high-power laser beams has opened new possibilities in the field of research. Laser beams can generate plasma from interaction with solid targets, and, in proper conditions, they can also trigger nuclear reactions. Thus, laser induced phenomena have acquired a great deal of attention from scientists working in a variety of areas like plasma physics and nuclear fusion [5]. For this reason, at LNS a dedicated laboratory was realized in order to investigate plasmas produced by laser-matter interaction and to better understand the nuclear process involved in the stellar burning.

\section{LENS Laboratory}

At LENS (Laser Energy for Nuclear Science) laboratory a Q-switched Nd:YAG commercial laser, produced by Quanta System, having a maximum energy of $2 \mathrm{~J}$, a fundamental wavelength of $1064 \mathrm{~nm}$, a duration of $6 \mathrm{~ns}$ and a repetition rate of $10 \mathrm{~Hz}$ is employed. The laser beam passes through an optical sampler that extracts about $1 \%$ of the primary beam and conveys it onto a pyroelectric energy meter in order to measure the energy of each pulse. The laser pulse is focused onto a flat target of aluminium by means of

* Corresponding author: altana@lns.infn.it 
a plano-convex lens. The interaction with the target occurs inside a vacuum chamber, where a pressure of about $10^{-5}$ mbar. The laser beam impinges on the target with an angle of $30^{\circ}$ with respect to the normal. The best focusing condition of the laser beam on the target is obtained by varying the lens-target distance in order to maximize the soft X-ray yield. The total intensity reached was in the range $10^{12}-10^{13} \mathrm{~W} / \mathrm{cm}^{2}$. The vacuum chamber is cylindrical stainless-steel tube with diameter $80 \mathrm{~cm}$ and is provided with appropriate windows for laser beam entrance and light collection. A schematic representation of the vacuum chamber and relative diagnostics is shown in Fig. 1.

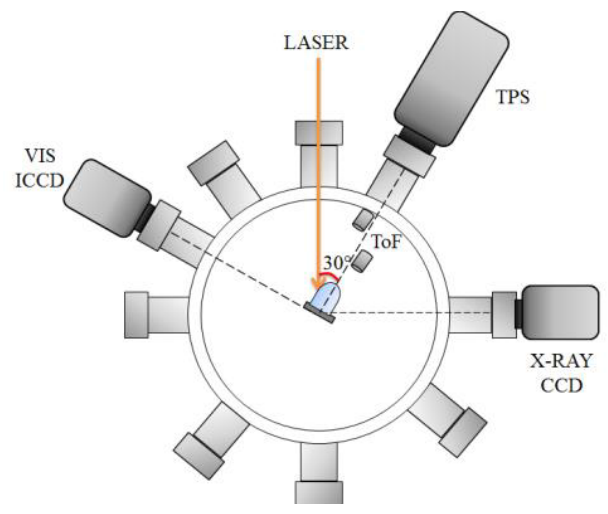

Fig. 1. Schematic view of the experimental setup.

A metal target holder has been employed to keep the target in position and to connect it to an externally controlled X-Y motor. In fact, in order to avoid errors due to local heating and drilling, traslation along $\mathrm{x}$ and $\mathrm{y}$-axis is needed to provide fresh surface for each shot. Also the focal lens is equipped with a motor along the laser direction (z-axis), which allows to change the distance between the lens and the target in order to find the focus position. Several detectors for visible, X-ray radiation and ionic component studies are connected to the vacuum chamber [6-8]. All the diagnostic is equipped with an external trigger signal, which is synchronized with the beginning of the laser pulse.

\subsection{Visible photons, $\mathrm{X}$-ray and lonic component characterization}

The detector used for the optical fast imaging is an Intensified Charged Coupled Device (ICCD), comprised a gated intensifier coupled to a front illuminated (CCD) camera via a high-quality relay lens system, which was supplied by Andor Technology [2]. The CCD chip consist in $1024 \times 1024$ pixels with a pixel size of $13 \mu \mathrm{m}$ and an effective active area of $13.3 \times 13.3 \mathrm{~mm}^{2}$. The Image Intensifier tube is an evacuated tube, which comprises a Photocathode, a Microchannel plate (MCP) and a Phosphor screen. The ICCD is equipped with an internal Digital Delay Generator (DDG), which allows to track over time the images in order to extract important information such as their spatio-temporal distributions and velocities. The bright plasma emission was observed through a window perpendicularly placed to the plasma expansion direction, with a magnification of 1.3 on the detection plane.

The CCD camera, by Andor Technology, consists of a $26.7 \times 6.7 \mathrm{~mm}^{2}$ CCD device with $1024 \times 256 \mathrm{X}$-ray sensitive silicon pixels, allowing the detection of X-ray photons. Each pixel is $26 \mu \mathrm{m}$ size and it is back illuminated; in this way the absorption of X-rays in the low-energy domain is minimized. The CCD can be cooled down to a temperature of $100{ }^{\circ} \mathrm{C}$ (under vacuum) obtaining a strong reduction of the dark-signal. Half of the sensitive surface is used to measure the total X-ray flux by means of a pinhole array; each pinhole 
was filtered to be sensitive to a different spectral region by using Al-made attenuators of increasing thicknesses, ranging from 3 to $20 \mu \mathrm{m}$. The other half of the sensitive surface is covered by a Ti filter in order to operate in a Single Photon regime, allowing to measure the energy spectrum.

Time of Flight - Ion collectors are placed inside the chamber at different distances, at approximately $15 \mathrm{~cm}$ and $30 \mathrm{~cm}$ from the target, to about $5^{\circ}$ respect to the plasma direction propagation. Ions collectors allow to calculate the velocity distribution of charged particles escaped from the plasma. Recently has been implemented new solid state detectors base on Silicon Carbide material [10-14], which can be used as e plasma diagnostic and also as nuclear fragments detectors. Moreover, a Thomson Parabola Spectrometer (TPS) is employed to characterize ion beams. The working principle [15-17] is based on parallel electric and magnetic fields acting on a well-collimated ion beam propagating orthogonally to the fields themselves. The combined effect of electric and magnetic fields deflects the different ion species on parabolas with different charge state, while a single parabola is marked from ion with the same charge state but different energy. After passing the drift region, which allows to increase the particles deflection and separation among different traces, the ions position is detected using an imaging system. In our case, a microchannel plate coupled to a phosphor screen and a reflex camera has been used to acquire the produced light.

\section{Results}

Fast photography in visible domain is one of the versatile diagnostic tools for understanding the expansion dynamics of laser created plasmas, that gives two-dimensional snap shots of the plume propagation, as shown in Fig. 2.
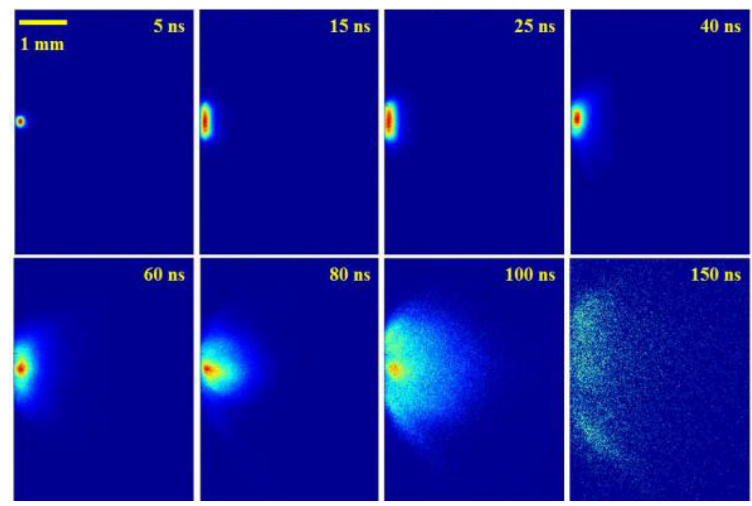

Fig. 2. Time sequence of fast photography images showing the spatio-temporal evolution of the plume at various delays.

It is possible to study the expansion velocity of the visible plasma plume from fit of the R-t plot, where $\mathrm{R}$ is the distance of the luminous front (corresponding to the point in which the maximum intensity is reduced by $90 \%$ ), and $t$ is the time instant of the snapshot. From the slope of the linear fit, an average plasma expansion velocity of the order of $1 \times 10^{4}$ $\pm 0.2 \mathrm{~m} / \mathrm{s}$ was evaluated, according to literature values [18].

From energy spectrum in X-ray region it is possible to estimate the average, space-time integrated, plasma temperature. A temperature of about $150 \mathrm{eV}$ was calculated, assuming ionic recombination and bremsstrahlung as the main emission sources [19]. 
In time of flight measurements, average speeds of the ionic component of the order of $3 \times 10^{5} \mathrm{~m} / \mathrm{s}$ are obtained. While, from TPS analysis, it can be observed that the plasma is composed of different charge states of aluminium and protons (due to surface impurities). Maximum energies respectively of aluminium and protons are estimated to be $30 \mathrm{keV}$ and 5 $\mathrm{keV}[20-21]$.

\section{References}

1. C. Spitaleri et al., Phys. Rev. 95, Issue:3, 035801 (2017)

2. M. La Cognata et al., Phys. Lett. B 664, Issue:3, 157 (2008)

3. A. Tumino et al., Phys. Lett. B 750, 59 (2015)

4. D. Mascali et al., Rad. Eff. And Defec. in Solids 165, Issue:6, 730 (2010)

5. F. Negoita et al., Romania Reports in Phys. 68, S37 (2016)

6. S. Privitera et al., Sensors 8, Issue:8, 4636 (2008)

7. D. Mascali et al., Eur. Phys. Lett. 100, Issue:4, 45003 (2012)

8. S. Tudisco et. al., Nucl. Instr. \& Meth. A 653, Issue:1, 47 (2011)

9. https://andor.oxinst.com

10. A. Muoio et al., EPJ Web of Conferences 117, 10006 (2016)

11. S. Tudisco et al., Sensors 18, Issue:7, 2289 (2018)

12. C. Ciampi et al. Nucl. Instr. \& Meth. A 925, 60 (2019)

13. S. Nida et al., Jour. of Synch Rad. 26, 28 (2019)

14. M. Rebai et al., Nucl. Instr. \& Meth. A 946, 162637 (2019)

15. M. J. Rhee, Rev. Sci. Instrum., 55.8 (1984)

16. F. Schillaci et al., Jour. of Phys. Conf. 508, Issue:1, 012010 (2014)

17. G.A.P. Cirrone et al. Proc. of SPIE 8779, 87791 (2013)

18. N. Farid et al., Physics of Plasmas, 20(7), 073114 (2013)

19. D. Mascali et al., Rev Sci Instrum 85 (2014)

20. S. Tudisco et al., Rev Sci Instrum., 87 (2016)

21. N. Gambino et al., Appl. Surf. Scie. 272, 69 (2013) 